\title{
Optofluidic Distributed Feedback Dye Lasers
}

\author{
Zhenyu Li, Student Member, IEEE, and Demetri Psaltis, Fellow, IEEE
}

\author{
(Invited Paper)
}

\begin{abstract}
We review our recent work on poly(dimethylsiloxane) (PDMS)-based optofluidic dye lasers using a guided wave distributed feedback (DFB) cavity. We show experimental results of single-mode operation, an integrated laser array, multiple color dye lasing, mechanical and fluidic tuning, and monolithic integration with microfluidic circuits. Potential applications and future directions are discussed.
\end{abstract}

Index Terms-Distributed feedback (DFB) lasers, dye lasers, lab-on-a-chip, microfluidic integration, optofluidics, single-mode lasers, tunable lasers.

\section{INTRODUCTION}

$\mathbf{T}$ HE motivation for building liquid dye lasers on a microfluidic chip is straightforward: with an on-chip widely tunable laser source, many fluorescence and spectroscopy-based techniques can be fully integrated with microfluidics to build labon-a-chip systems. The emergence of microfluidics has made it possible to realize a variety of optical functionalities using the fluidic platform [1]. A miniature dye laser is one of the most important examples of such optofluidic devices, in which the integration of microfluidics with the adaptive nature of liquids enables unique performance that is not obtainable within solid state materials. The dye lasers that will be described in this paper are implemented with soft lithography techniques [2], [3]. In particular, we have used the poly(dimethylsiloxane) (PDMS) silicone elastomer, even though any other optically transparent soft elastomer can also be used. Using replica molding soft lithography, microfluidic chips can now be routinely made of PDMS in less than a few hours once the master mold is at hand. The mask design and mold fabrication can also be quickly done and the resulting mold can be used multiple times. In addition, with multilayer soft lithography, active pressure-driven mechanical microvalves and pumps can be made using a double-layer crossed-channel architecture [3]. Such valves and pumps enable very high degree of microfluidic automation [4] and large-scale integration [5], making on-chip picoliter-scale liquid handling fast and accurate.

In the past few years, several groups have demonstrated onchip liquid dye lasers [6]-[8], [13]. Here, we will focus on our demonstration of an optofluidic distributed feedback (DFB) dye laser that permits single-mode operation and wide tunabil-

Manuscript received October 2, 2006; revised February 14, 2007. This work was supported by the Defense Advanced Research Projects Agency (DARPA) Center for Optofluidic Integration.

$\mathrm{Z}$. Li is with the Department of Electrical Engineering, California Institute of Technology, Pasadena, CA 91125 USA (e-mail: zhenyu@caltech.edu).

D. Psaltis is with the Defense Advanced Research Projects Agency (DARPA) Center for Optofluidic Integration, California Institute of Technology, Pasadena, CA 91125 USA, and also with Ondax, Inc., Monrovia, CA 91016 USA (e-mail: psaltis@sunoptics.caltech.edu).

Digital Object Identifier 10.1109/JSTQE.2007.894051

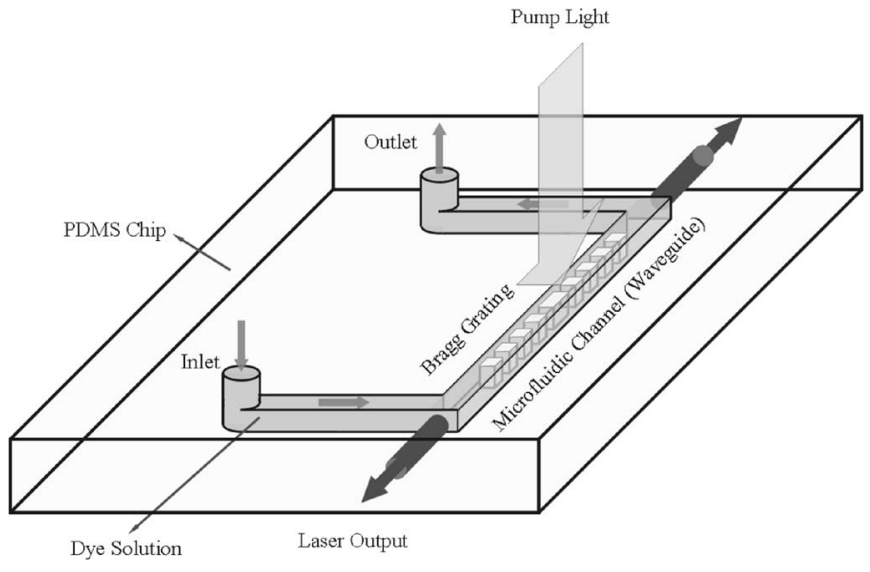

Fig. 1. Schematic diagram of the monolithic optofluidic DFB dye laser.

ity [9], [10]. The paper is organized as follows: Section II describes a single-mode optofluidic DFB dye laser on a monolithic PDMS chip, including the cavity design, the fabrication, and the experimental results. Section III presents the mechanical wavelength tuning method of such lasers exploring the soft elastomer nature of PDMS. Section IV discusses the automated fluidic tuning of the laser wavelength and the integration with microfluidic circuits. Section V gives the experimental results of an integrated DFB dye laser array and its potential applications. Section VI points out some future directions. Section VII summarizes the paper.

\section{Single-Mode Optofluidic DFB Dye LASER ON A MONOLITHIC PDMS CHIP}

\section{A. Laser Chip Design}

After the first demonstration of a DFB dye laser at Bell Labs in 1971 [11], DFB structures were quickly adopted by the semiconductor laser community [12]. One reason is that the DFB structure is very efficient for making stable single-frequency lasers, which are important for long-distance communication applications. The other is that the DFB structure is amenable to planar semiconductor processing techniques. Now, with the development in soft lithography, very similar structures to that of a guided-wave DFB semiconductor laser can be built on a PDMS microfluidic chip with liquid dye gain medium [9]. As shown in Fig. 1, a sufficiently small microfluidic channel when filled with a dye solution of higher refractive index than that of PDMS $\left(n_{\text {PDMS }}=1.406\right.$ for GE RTV 615) acts as a single-mode optical waveguide. The gain medium is a dye solution in an organic solvent mixture with higher refractive index. The periodic PDMS posts inside the channel form a Bragg grating that provides the 
optical feedback necessary for the laser action. In addition, an effective $\pi / 2$ phase shift is introduced at the center of the grating to ensure single-frequency operation [14]. The PDMS posts also provide support for the microfluidic channel to prevent it from collapsing. Nanosecond pulses from a Q-switched Nd:YAG laser are focused onto the channel to pump the dye molecules. The laser light is emitted from the edge of the channel.

Such microfabricated dye lasers retain the advantages of conventional dye lasers such as wide wavelength tunability, narrow linewidth, and the ability to generate ultrashort pulses [15]. But probably, the most important feature is its capability of integration with PDMS-based microfluidics, which allows us to build not only fully functional lab-on-a-chip systems such as biosensors and spectrometers on a chip, but also novel adaptive optofluidic dye laser systems in which the laser parameters such as wavelength, spatial mode, and temporal features are fluidically modified. In addition, such microfabricated lasers consume only picoliter-scale dye solutions while the low fabrication cost makes disposable devices possible. The remaining open issue for making fully integrated devices is the integration of the optical pump onto the same chip. The key feature that will allow us to do this is a reduction in the lasing threshold, which will be discussed later in this paper.

\section{B. Longitudinal Mode Selection}

A DFB structure uses a Bragg grating, which is a spatially periodic modulation of refractive index (or gain). When the following Bragg condition is satisfied

$$
m \lambda=2 n_{\mathrm{eff}} \Lambda
$$

where $\lambda$ is the $m$ th-order resonant wavelength, $n_{\text {eff }}$ is the effective index of the guided mode, and $\Lambda$ is the grating period, which means the round trip optical path of one grating period equals integral multiples of the wavelength, and all the reflections from the unit cells will add up in phase. Compared with ordinary Fabry-Perot lasers, DFB lasers offer a much better wavelength stability. Normally, a DFB laser uses a first-order Bragg grating for efficiency and compactness of the device. However, fabrication of subwavelength gratings and small channels in soft materials such as PDMS represents a technical challenge because small channels tend to collapse more easily when the aspect ratio (the ratio of channel height to width) is small, and small grating features tend to contact each other when the aspect ratio is large [2]. Therefore, our initial efforts focused on higher order DFB structures. In order to achieve single longitudinalmode operation, the free spectral range (FSR) of the optical cavity must be larger than the gain spectral width of the laser medium. The FSR of a DFB structure is given by

$$
\mathrm{FSR}=\lambda^{2} / 2 n_{\mathrm{g}} \Lambda
$$

where $n_{g}$ is the group index of the mode. Given the gain bandwidth of the dye medium is typically about $30 \mathrm{~nm}$, the grating period needs to be smaller than $4 \mu \mathrm{m}$ when the refractive index is about 1.4, which is a typical value for liquids. In addition, if the shape of the grating is a square-wave with $50 \%$ duty cycle, all the even-order resonances will disappear. Thus, a 4- $\mu \mathrm{m}$ period Bragg grating can have an effective FSR of $\sim 60 \mathrm{~nm}$.

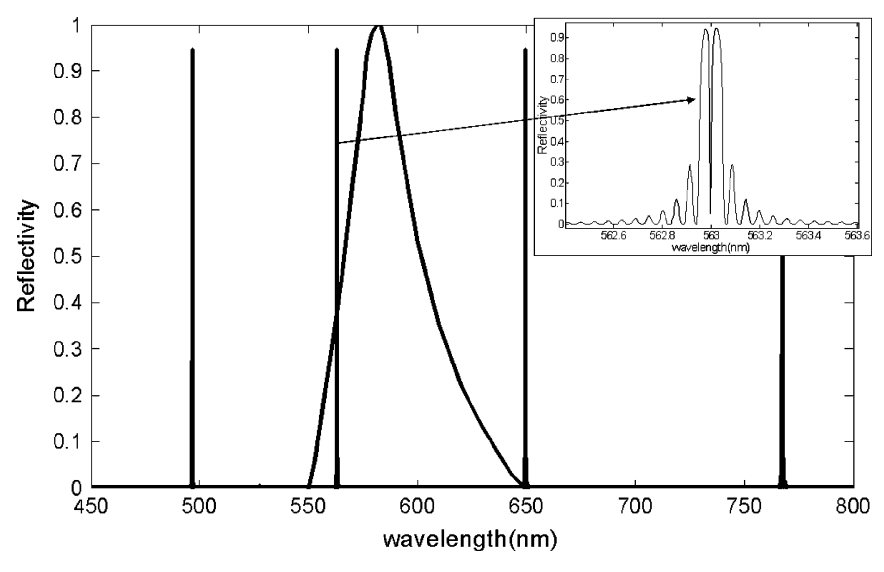

Fig. 2. Simulated reflectivity spectrum of a $15 \pi / 2$ phase-shifted 15 th-order DFB structure. The curve spanning from 550 to $650 \mathrm{~nm}$ is the gain spectrum of Rhodamine 6G. The inset shows the enlarged plot of the 15th resonance at $563 \mathrm{~nm}$.

If the grating period is $3 \mu \mathrm{m}$, the corresponding Bragg order is 15 at $563 \mathrm{~nm}$. Fig. 2 shows the simulated reflectivity spectrum using Rouard's method [16]. The index difference between the core and the cladding is 0.003 . The core size is $2 \mu \mathrm{m} \times 5 \mu \mathrm{m}$. These waveguide parameters are chosen to ensure single transverse mode as discussed later in Section II-C. Also shown in Fig. 2 is the gain spectrum of the Rhodamine 6G (Rh6G) dye molecule. As can be seen, there is only one resonance under the whole gain spectrum. However, it is well known that for a DFB laser with a uniform grating, there are two degenerate lasing modes with comparable thresholds. One way to solve this problem is to introduce a $\pi / 2$ phase slip at the center of the grating to break this degeneracy [14]. In our case, we introduce a $15 \pi / 2$ phase shift, which is compatible with the 15 th-order grating structure and the fabrication method. Also, from this spectrum, we can see this structure has three resonances in the visible region, which means if we use different dyes we can achieve different color lasing from the same cavity. We show in Section III the experimental results of yellow and red lasing on the same chip using two dye solutions, Rh6G and Rhodamine 101 (Rh101) in a mixture of methanol and ethylene glycol. This is a useful feature, which, we believe, has not been explored in semiconductor DFB lasers, since the gain spectrum of a single semiconductor material is limited. However, with dye lasers we can easily move across the whole visible region by changing dye molecules. This is a highly desired feature for applications where multiwavelength laser sources are needed such as multicolor flow cytometry [17] or fluorescence resonance energy transfer (FRET) with multiple donors and acceptors. With a mixture of several dye molecules, simultaneous multiple-color lasing is also possible. However, compared with the first-order DFB structure, higher order DFB cavities are less efficient in terms of light confinement because the coupling coefficient is inversely proportional to the order of the grating [18]. Moreover, out-of-plane radiation due to lower order scattering increases the cavity loss [19]. This can be compensated to some degree by increasing the cavity length to provide strong-enough feedback. 
TABLE I

REFRACTIVE INDICES OF PDMS-COMPATIBLE SOLVENTS

\begin{tabular}{|l|l|}
\hline \hline \multicolumn{1}{|c|}{ Solvent } & \multicolumn{2}{|c|}{ Refractive Index $^{\mathrm{a}}$} \\
\hline Ethanol & 1.36 \\
\hline Methanol & 1.33 \\
\hline DI water & 1.333 \\
\hline Ethylene Glycol & 1.43 \\
\hline DMSO & 1.478 \\
\hline
\end{tabular}

${ }^{\mathrm{a}}$ At room temperature.

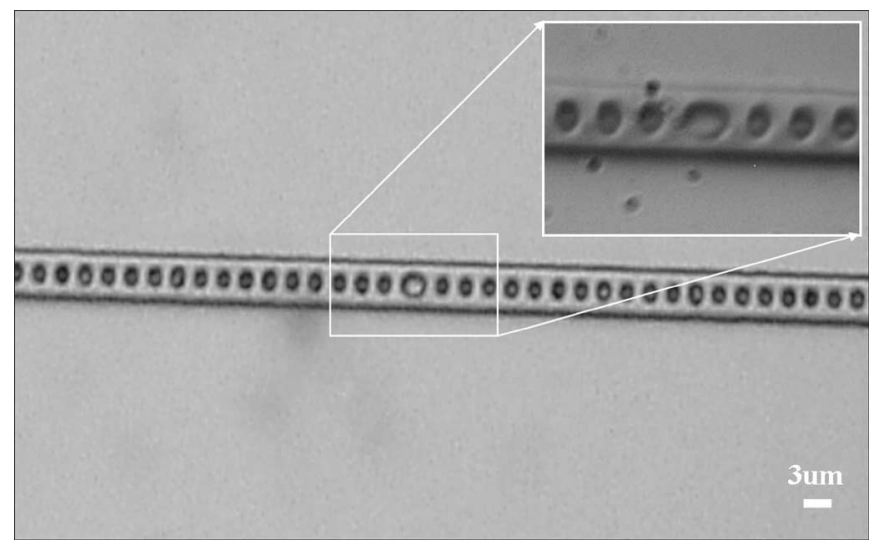

Fig. 3. Optical micrograph of a microfluidic channel with a 15th-order DFB structure on a PDMS chip. The grating period is $3 \mu \mathrm{m}$.

\section{Transverse-Mode Selection}

To make a single-mode laser, the transverse-mode control must also be considered. The number of transverse modes is determined by the index contrast between the waveguide core and the cladding and the size of the core. We choose the waveguide to be 5- $\mu \mathrm{m}$ wide and $2-\mu \mathrm{m}$ high. The reasons for this are twofold: first, the waveguide dimensions are relatively small, yet still easily achievable by photolithography; second, the $2: 5$ aspect ratio is large enough so that the PDMS channel will not collapse. For a channel waveguide with a $2 \mu \mathrm{m} \times 5 \mu \mathrm{m}$ core, finite-element simulation (FEMLAB 3.2, Comsol) shows that the index difference needs to be smaller than 0.003 to maintain single mode. Given the index of PDMS (1.406 for GE RTV 615), this means the index of the dye solution has to be smaller than 1.409. This can be specified by mixing two different organic solvents. The choice of solvents is limited due to the swelling problem of PDMS in most organic solvents [20]. The PDMS-compatible solvents which are also used in conventional dye lasers include: ethanol, methanol, water, ethylene glycol, and dimethylsulfoxide (DMSO), the refractive indices of which are listed in Table I. We used a mixture of methanol and ethylene glycol, which has a refractive index of 1.409. The available refractive index range with the listed solvents is from 1.33 to 1.478 , which can lead to a quite a large tuning range when used in fluidically-tunable optical devices.

\section{Fabrication and Experimental Results}

Fig. 3 shows an optical micrograph of the fabricated PDMS DFB laser cavity. We started the fabrication by making a patterned photoresist mold on a silicon wafer using conventional

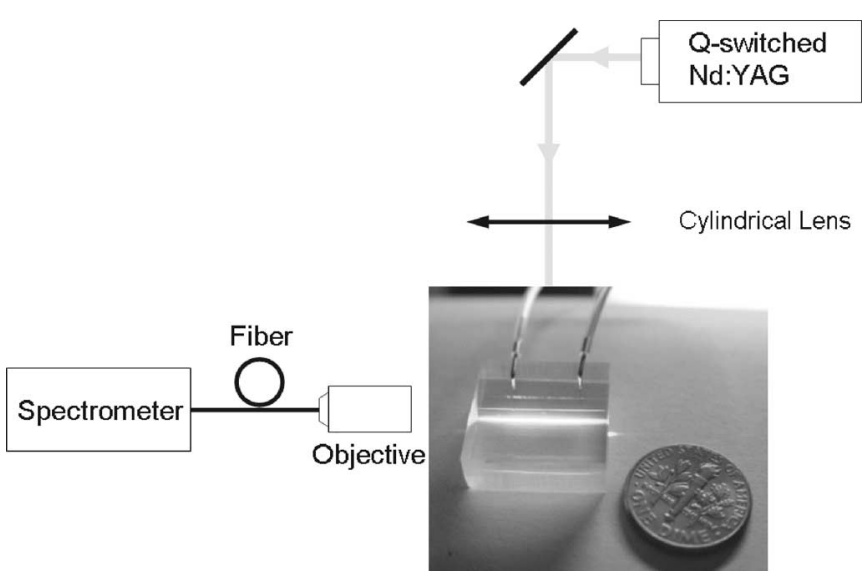

Fig. 4. Experimental setup used for characterizing the optofluidic DFB dye laser.

photolithography. SU8-2002 negative photoresist (MicroChem) was spin-coated at $3000 \mathrm{r} / \mathrm{min}$ for $30 \mathrm{~s}$ on a silicon wafer yielding a thickness of $\sim 2 \mu \mathrm{m}$. The photoresist was then patterned with a Cr-on-glass mask on a SUSS MJB 3 mask aligner $\left(13 \mathrm{~s}\right.$ at $\left.4.5 \mathrm{~mW} / \mathrm{cm}^{2}\right)$. The soft bake, postexposure bake, and development of SU8 followed the manufacturer's instructions. Then, GE RTV 615 prepolymer at a ratio of 5:1 part A:B was poured onto the mold and degassed in a vacuum chamber for $30 \mathrm{~min}$. The prepolymer was baked in an $80^{\circ} \mathrm{C}$ oven for 30 min. The partially cured PDMS was peeled from the mold and the access ports were punched through the whole layer by using a 0.055 -in punch (Technical Innovations, Gaithersburg, MD). This patterned PDMS, containing the laser structure, was then treated with oxygen plasma for $10 \mathrm{~s}$ at $80 \mathrm{~W}$ and bonded to another featureless PDMS to form a permanent monolithic device. Finally, the resulting device was baked at $80^{\circ} \mathrm{C}$ overnight.

The experimental setup is shown in Fig. 4. The laser chip was optically pumped with 6-ns frequency-doubled Q-switched Nd:YAG laser pulses of 532-nm wavelength, focused by a cylindrical lens of $10-\mathrm{cm}$ focal length to a $\sim 100-\mu \mathrm{m}$ wide stripe covering the whole microfluidic channel. A $10 \times$ microscope objective was used to collect the emission light from one edge of the chip and deliver it to a fiber-coupled high-resolution spectrometer with 0.1-nm resolution (Ocean Optics HR4000). The 1-mM Rh6G dye solution was pressure driven into the microfluidic channel by applying 10 psi pressure at the inlet port. A typical single-mode lasing spectrum is shown in Fig. 5. The broad curve is the spontaneous emission spectrum below the threshold. Above the threshold, the emission spectrum collapsed to a sharp line centered at $570 \mathrm{~nm}$, very close to the predicted value $570.3 \mathrm{~nm}$ for the grating period $3.04 \mu \mathrm{m}$. The measured linewidth is less than $0.1 \mathrm{~nm}$, which is the resolution limited by the spectrometer. A plot of laser output energy versus the absorbed pump energy is shown as the inset of Fig. 5. The threshold pump fluence is estimated to be $\sim 0.8 \mathrm{~mJ} / \mathrm{cm}^{2}$ taking into account the dye absorption, dye layer thickness, interface reflections, and the total pump area, which gives a peak pump intensity around $150 \mathrm{~kW} / \mathrm{cm}^{2}$. The laser remains single mode at pump levels as high as $8 \mathrm{~mJ} / \mathrm{cm}^{2}$. Further increase in the pump 


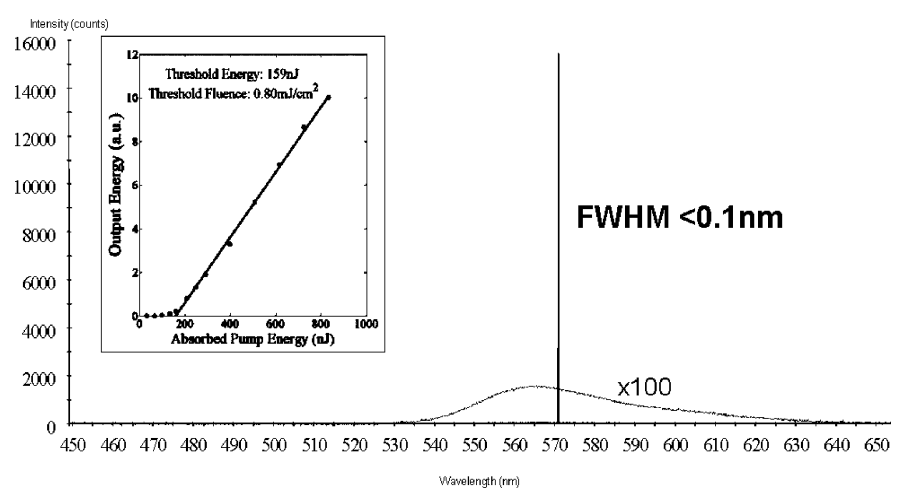

Fig. 5. Optofluidic DFB dye laser spectrum. The measured linewidth is $<0.1 \mathrm{~nm}$. The inset shows the output energy versus the absorbed pump energy curve. The threshold pump fluence is $\sim 0.8 \mathrm{~mJ} / \mathrm{cm}^{2}$.

power damaged the PDMS chip. At moderate pump intensities $\left(\sim 200 \mathrm{~kW} / \mathrm{cm}^{2}\right)$ and $10-\mathrm{Hz}$ repetition rate, stable laser output lasted longer than $20 \mathrm{~min}$ and the chip can be reused many times after cleaning with ethanol. The linear flow rate at the center of the channel can reach $1 \mathrm{~mm} / \mathrm{s}$ at reasonable pressure (10-30 psi). Considering the typical photobleaching time of Rh6G is $\sim 50 \mathrm{~ms}$ under pump intensities required for laser threshold $\left(\sim 100 \mathrm{~kW} / \mathrm{cm}^{2}\right)[21]$, this flow rate is fast enough to prevent the majority of the dye molecules from photobleaching under pulse operation up to $500 \mathrm{kHz}$. However, the dye molecules near the channel wall move at very low velocities due to the parabolic profile of the flow rate. Therefore, they tend to get bleached before moving out of the pump region. This problem can be solved by using a liquid core/liquid cladding waveguide structure in which the dye solution is surrounded by a low refractive index sheath flow [22]. In this way, not only the dye solution is moving at a more uniform speed, but also the channel dimensions can be large enough to enable higher flow rate.

We have measured laser output energy $>1 \mathrm{pJ}$ per pulse without damaging the chip. Assuming a 10-ns pulse width, this corresponds to an average power of $\sim 100 \mu \mathrm{W}$ per pulse and an average intensity of $\sim 1 \mathrm{~kW} / \mathrm{cm}^{2}$. This intensity is high enough for many fluorescence and even spectroscopy applications. In addition, the current device is not designed for high power output. If higher power is desired, the transverse waveguide dimensions can be increased and a more efficient pump geometry can be used.

\section{ElAStiC TUNING OF LASING WAVELENGTH}

The most attractive feature of dye lasers is their wide tunability. Therefore, it is natural to study the various tuning methods of the optofluidic DFB dye laser. The lasing wavelength of a DFB laser can be tuned by changing either $n_{\mathrm{eff}}, \Lambda$, or $m$ as has been demonstrated in conventional dye lasers [23]. In this section, we first discuss a very simple way to change the grating period $\Lambda[10]$.

The fact that PDMS is an optically transparent (300-800 nm) soft elastomer has been explored to make adaptive optical elements, or the so-called elstomeric optics in which the stretching, compressing, and bending of the elastomeric material modulates the optical properties such as the period of a diffraction grating, the focal length of a lens, and the curvature of a mirror [24]. The advantages of using PDMS include: 1) it can be deformed under small forces due to its very low Young's modulus ( $\sim 750 \mathrm{kPa})[25] ; 2)$ its elastomeric property makes the deformation reversible and repeatable without permanent distortion; 3) it can be molded at a subwavelength scale and with optically smooth surfaces; 4) it is biocompatible and microfluidics compatible; and 5) the material and fabrication cost is low.

In the context of PDMS optofluidic DFB dye lasers, the Bragg grating period can be tuned by simply stretching or compressing the chip along the waveguide direction since the laser cavity is made of a liquid medium within an elastomer container. The wavelength tuning range of a DFB laser is proportional to the fractional change in the grating period, $d \lambda / \lambda=d \Lambda / \Lambda$. Given that the allowed elongation of PDMS is $>120 \%$ [26], the wavelength tuning range is only limited by the gain bandwidth of the specific dye. By combining mechanical tuning and switching between two different dye solutions, we have demonstrated a nearly 60-nm single-mode tuning range from yellow to red.

The wavelength tuning experiments were carried out using the same laser chip as in Section II. The PDMS chip was glued to two micrometer stages with the laser region suspended in the center. One of the stages is a high-resolution micrometer with $1-\mu \mathrm{m}$ sensitivity which provides accurate control and quantitative measurement of the deformation of the elastomer chip. The mechanical stages allow us to both stretch and compress the chip along the channel direction, although the allowed shrinkage of RTV 615 is very limited [26]. The results of mechanical cavity tuning are summarized in Fig. 6 . The single-mode tuning range for Rh6G covers a range from 565 to $594 \mathrm{~nm}$, whereas for $\mathrm{Rh} 101$, the tuning range extends from 613 to $638 \mathrm{~nm}$. A linear relationship between the lasing wavelength and the chip deformation was also observed, indicating complete elastic extension of the cavity. When the length of the central suspended region is 1 $\mathrm{cm}$, the total chip deformation required to traverse the previously mentioned tuning ranges are about $500 \mu \mathrm{m}$ for Rh6G and 400 $\mu \mathrm{m}$ for Rh101. These macroscopic deformations correspond to 28-nm- and 25-nm distortions of the grating period, respectively. Only $\sim 5 \%$ deformation was used to achieve the $\sim 60$-nm tuning range demonstrated in this experiment, and we believe that an even wider tuning range from 550 to $650 \mathrm{~nm}$ is obtainable with improved cavity design and a more uniform mechanical loading. The tuning is continuous and completely reversible, and no noticeable degradation of the chip was observed during a 100-cycle full range tuning test. Throughout the tuning range, stable singlemode operation was maintained with measured linewidth below $0.1 \mathrm{~nm}$, which is the resolution limited by our spectrometer. As expected, we observed the decrease of laser output power as the lasing wavelength moved away from the gain spectrum peak in either direction. The deformation along the channel also causes its transverse dimensions to change, which changes the effective index of the guided mode. However, given that the Poisson's ratio of PDMS is $\sim 0.5$, the estimated effective index change is only about $1.5 \times 10^{-5}$ and its effect on the lasing wavelength is negligible. 

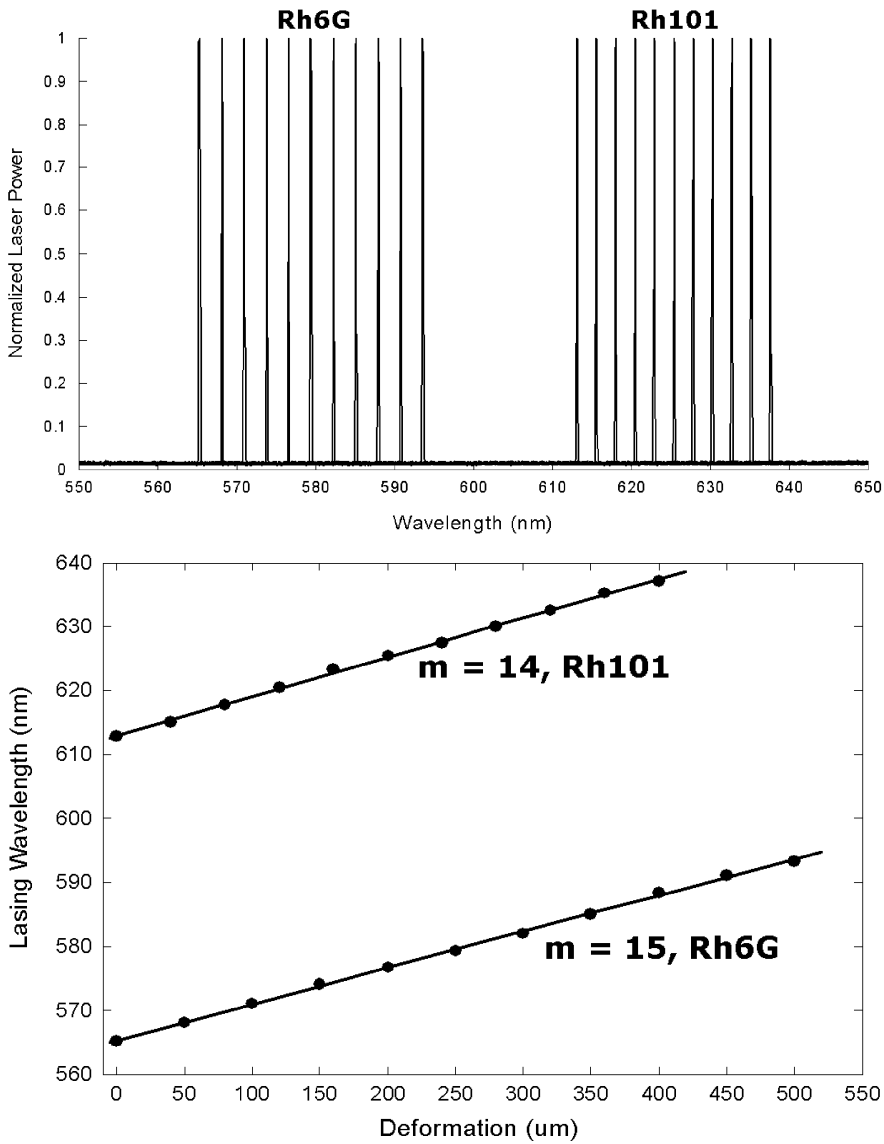

Fig. 6. Upper: Normalized laser output of the mechanically-tunable optofluidic DFB dye laser. Different peaks correspond to different grating periods. The measured laser linewidth is less than $0.1 \mathrm{~nm}$ throughout the tuning range. Lower: Lasing wavelength versus the measured chip deformation. The points are the experimental data and the curve is the linear fit. The achieved singlemode tuning range for Rh6G is from 565 to $594 \mathrm{~nm}$ and is from 613 to $638 \mathrm{~nm}$ for Rh101.

In some cases, the transverse loading geometry may be more preferred, in which case the deformation force is applied transverse to the channel. Since PDMS is almost a perfectly incompressible material with Poisson's ratio $\sim 0.5$, the transverse strain can be efficiently translated to longitudinal strain. We have also implemented elastic tuning using a transverse load and achieved a $\sim 10$-nm tuning range for each dye mentioned earlier.

\section{Fluidic Tuning of LASing WaVelength}

The microfluidics compatibility of the laser chip suggests another wavelength tuning mechanism. The refractive index of the dye solution can be tuned by mixing two solvents with different refractive indices. For example, using methanol and DMSO, the achievable refractive index change can be as large as 0.148 ( 1.33 for methanol versus 1.478 for DMSO). Furthermore, as demonstrated earlier, different dye molecules can be used to cover an even larger spectral range. The mixing, switching, and transport of dye solutions can all be implemented on a silicone elastomer chip using the recently developed mechanical microvalves and pumps [3].

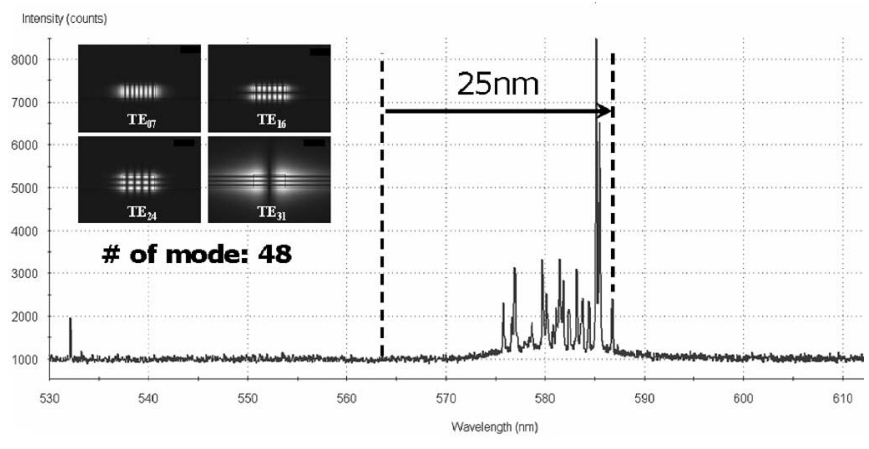

Fig. 7. Lasing spectrum of the optofluidic DFB dye laser with high index contrast between the liquid core and the PDMS cladding. The waveguide dimensions are $2 \mu \mathrm{m} \times 5 \mu \mathrm{m}$. The grating period is $3 \mu \mathrm{m}$. The refractive index of the Rh6G dye solution is 1.478 . More than 20 lasing modes were observed. The inset shows four simulated TE-like transverse-mode profiles.

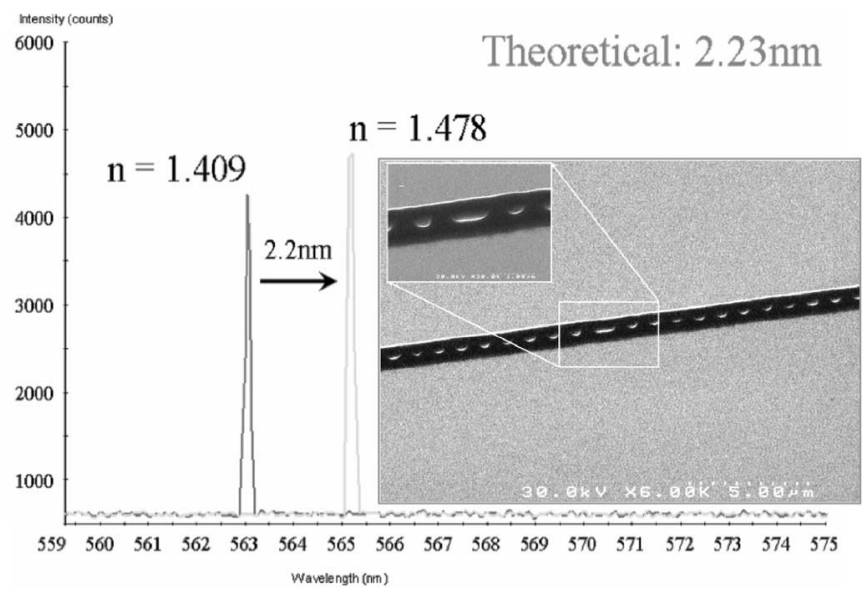

Fig. 8. Fluidic tuning of a 5th-order DFB dye laser with waveguide dimension $250 \mathrm{~nm} \times 880 \mathrm{~nm}$. The inset shows an SEM of the silicon mold. The grating period is $1 \mu \mathrm{m}$.

However, in the design discussed in Section II, the relatively large dimensions of the waveguide cause multiple transversemode operation when the index contrast is increased to above 0.003. Fig. 7 shows a measured lasing spectrum when a high refractive index dye solution is used. In this case, the gain medium is a solution of 2-mM Rh6G in DMSO, which has a refractive index of 1.478. In this experiment, the waveguide dimensions were $2 \mu \mathrm{m} \times 5 \mu \mathrm{m}$ and the grating period was $3 \mu \mathrm{m}$. More than 20 lasing lines were observed in the spectrum. Finite-element analysis shows the waveguide supports totally 48 transverse modes. The measured longest lasing wavelength was $587 \mathrm{~nm}$, close to the predicted $588 \mathrm{~nm}$ for the lowest $\mathrm{TE}_{00}$ mode, which had moved by $\sim 25 \mathrm{~nm}$ to longer wavelength compared with the case when the core index is 1.409 .

To avoid multiple transverse-mode operation during fluidic tuning, a smaller channel has to be used. We have fabricated a waveguide of transverse dimensions $250 \mathrm{~nm} \times 880 \mathrm{~nm}$ with an embedded 5th-order Bragg grating. The inset of Fig. 8 shows a scanning electron micrograph (SEM) of the master mold, which was a patterned silicon wafer fabricated using electron beam lithography and reactive ion etching (RIE). This waveguide 


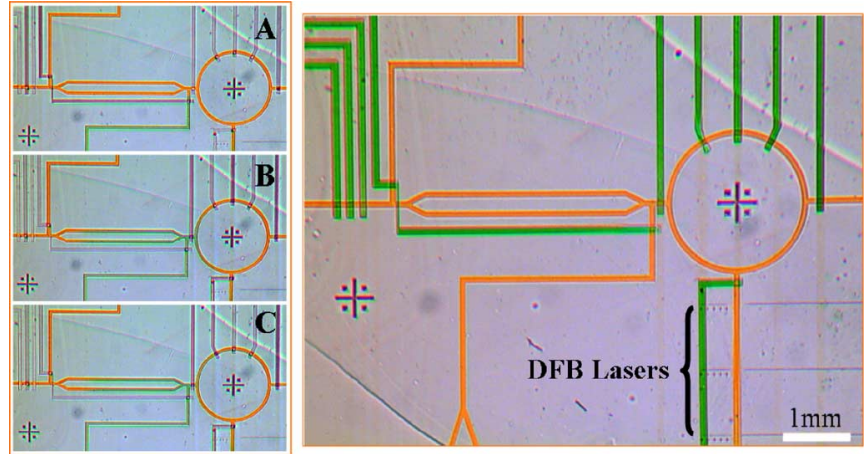

Fig. 9. Integration of a high-resolution microfluidic dispenser and a rotary mixer with DFB dye lasers. Left: Different stages of the microfluidic mixing. A. The rotary mixer is filled with orange dye solution; B. An accurate amount of green dye solution is delivered into the mixer; C. The pneumatic pump mixes the two solutions. Right: Optical micrograph of a monolithic PDMS device in which a microfluidic mixing circuit is integrated with an array of optofluidic DFB dye lasers.

remains single mode throughout the index range from 1.406 to 1.478 , which gives a predicted $2.23-\mathrm{nm}$ single-mode tuning range. The experimental lasing results matched the theoretical values almost exactly as shown in Fig. 8 .

The small tuning range is due to the small depth of the waveguide. The size of the guided mode is about $0.6 \mu \mathrm{m} \times 1 \mu \mathrm{m}$, which is considerably larger than the size of the waveguide core. Therefore, even a relatively large index change in the core results in only a small change in the effective index. The optimized waveguide geometry for large single-mode tuning range will be considered in a future work. Although this range is small compared to that of elastic tuning and the available gain bandwidth, better waveguide design and techniques such as Vernier effect [27] can be used to fully utilize the wide gain bandwidth of dye molecules.

A fully integrated microfluidic tunable DFB dye laser based on multilayer soft lithography is obtained by integrating a microfluidic mixing circuit with the dye laser cavity. Fig. 9 shows an automated high-resolution microfluidic dispenser and a rotary mixer integrated with an array of 5th-order DFB dye lasers. The upper green channels (dead-ended channels) are the control lines and the bottom orange layer is the fluid layer. The detailed operation of the dispenser and the mixer can be found in [4]. Accurate amount of two different dye solutions can be delivered into the mixer and these then get mixed uniformly before entering the laser cavities. All these manipulations can be computer controlled through a PCI card connected to a solenoid pressure valve array whose valves are connected to the control lines on the chip.

The fluidically-tunable DFB dye laser can also be used as a sensor to detect small refractive index or absorption changes in the liquid gain medium due to the presence of small amounts of certain analytes. The fluidic tuning can be also used to build tunable filters for fluorescence detection and spectroscopy applications. In many cases, fluidic tuning is preferred over solidstate-material-based methods due to its reconfigurable nature, higher index contrast $d n / n$, thermal stabilization, self-healing, optically smooth interfaces, ability to introduce active optical
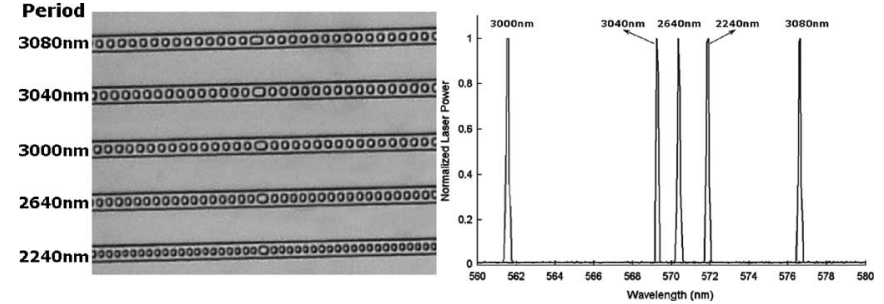

Fig. 10. Left: Optical micrograph of an integrated array of five optofluidic DFB dye lasers. The grating period of each laser is given on the left. Right: Normalized laser output of the array using Rh6G dye solution as the gain medium.

elements such as dyes, metal nanoparticles, and quantum dots, ability to generate gradients in optical properties, and the intrinsic compatibility with liquid-phase biological and chemical reactions [1].

\section{INTEGRATED LASER ARRAYS}

We have also fabricated an array of five DFB dye lasers on a single PDMS chip. Fig. 10 shows the array lasing results using a Rh6G dye solution. Each laser was pumped individually and the combined lasing spectrum was shown in Fig. 10. Laser output spanning a $\sim 15$-nm range was achieved with different grating periods. The low pump threshold $(<1 \mu \mathrm{J})$ of each optofluidic DFB dye laser makes it possible to use a single high-energy pulsed laser to pump hundreds of such lasers on a chip. This opens up the possibility of building highly parallel multiplexed biosensors on a chip ranging from applications as multiple-color flow cytometers and surface plasmon resonance (SPR)-based sensors to Raman spectroscopy sources and compact excitation spectroscopy systems. The introduction of replica-molded multispectral sources in PDMS fluidic systems also provides an alternative to tunable lasers for constructing compact and inexpensive multiwavelength scanning-less spectrometers integrated in microfluidic devices [28]. The low pump threshold also enables the use of visible semiconductor laser diodes or even high-power light-emitting diodes (LEDs) as the pump sources to construct low-cost and compact portable spectrometers.

A unique feature of soft lithography is the capability to bond multiple-patterned thin films of elastomer to build multilayer structures. Typically, a thick layer with the top structure is prepared as previously described. Each following thin layer of PDMS is spin-coated onto the mold with a typical thickness of $\sim 30 \mu \mathrm{m}$ and baked at $80^{\circ} \mathrm{C}$ for $20 \mathrm{~min}$. The growing thick layer is then assembled onto each new thin layer and bonded by oxygen plasma treatment and baking at $80^{\circ} \mathrm{C}$ for $20 \mathrm{~min}$. Seven-layer devices have been reported and no obvious limitations exist to limit the number of layers [3]. We have used this method to fabricate a three-layer DFB laser device with 10 lasers in each layer. Fig. 11 shows the optical microscope image of a two-layer DFB laser array. The slight misalignment between the two layers was deliberately introduced to make more efficient use of the total pump area. With multilayer devices, each layer can perform different functions so that the integration level and the compactness of the device can be greatly improved. 

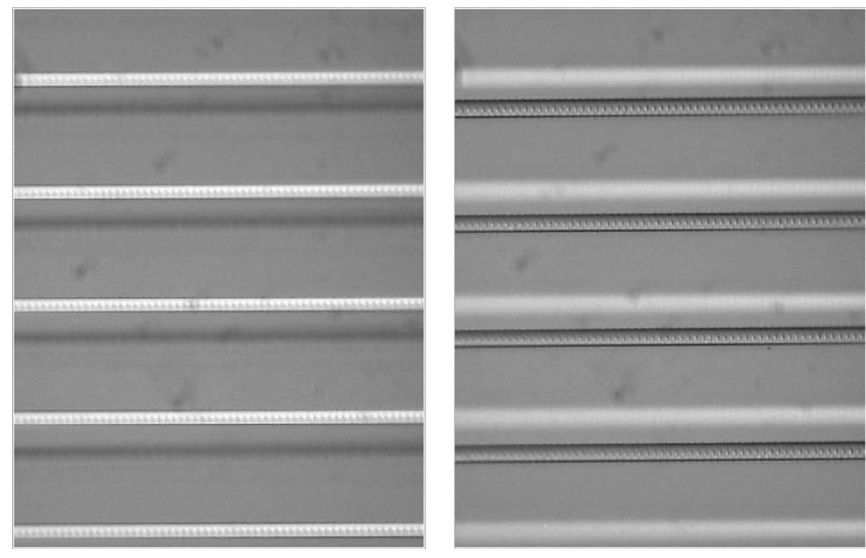

Fig. 11. Vertical stacking of optofluidic DFB laser arrays using multilayer soft lithography. Left: Upper laser array is in focus. Right: Bottom laser array is in focus.

\section{FUTURE DIRECTIONS}

Even though some impressive lasing features, such as singlemode operation, narrow linewidth and wide tunability, have been obtained, optofluidic dye laser research is still at its very early stage. In order for this technique to have an impact on the lab-on-a-chip systems, we believe the most important next step is to integrate the optical pump source onto the same laser chip. At present, due to the small gain volume, the required pump threshold intensity $\left(\sim 100 \mathrm{~kW} / \mathrm{cm}^{2}\right)$ is within the reach of commercially available high-power laser diodes. However, further improvement in pump efficiency can be achieved by introducing resonant structures at the pump wavelength or adapting a dual-core longitudinal pump geometry widely used in fiber lasers [29]. This may eventually lead to high-power LED pumped optofluidic dye lasers.

Another interesting direction is to implement other laser cavity structures beyond the currently demonstrated Fabry-Perot and DFB types onto the microfluidic platform, such as whispering gallery mode (WGM) resonators including microdisks [30], microrings [31], microtoroids [32], and microspheres [33], annular Bragg reflector (ABR) cavities [34], and photonic crystalbased cavities [35]. Moreover, in addition to provide novel laser cavity structures, the capability of building such liquid-core optical microcavities has implications in both fundamental study and practical applications. For example, such optofluidic microcavities should enable the study of cavity quantum electrodynamics (QED) effects, such as spontaneous emission engineering, thresholdless lasing, and single-photon sources, in a more controlled and reconfigurable manner, which, currently, is not available through solid state materials [36]-[38]. High Q and small-volume optofluidic microcavites are also ideal structures for single-molecule biological and chemical sensing. To demonstrate the feasibility of this direction, we show optofluidic microring lasing results obtained while we were working on the microfluidic tuning of DFB dye lasers. Recall that the microfluidic circuits we used to fluidically tune the lasing wavelength include a ring-shaped fluidic mixer, as shown in Figs. 9 and 12. When the pump light was focused on a small portion of the ring,

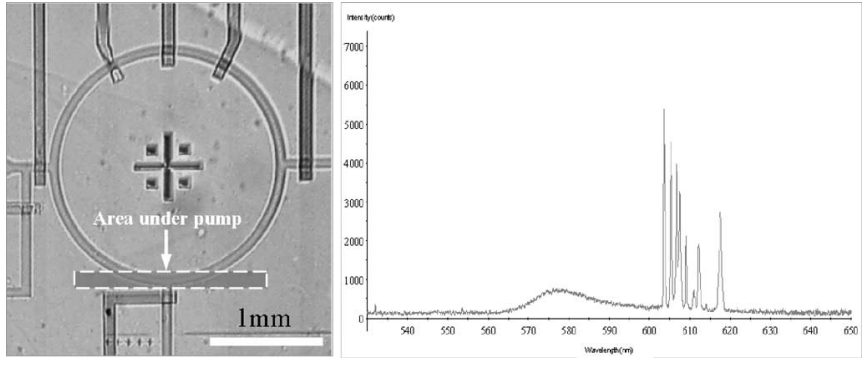

Fig. 12. Optofluidic microring dye laser. Left: A liquid core microring resonator initially used as a microfluidic mixer. The ring radius is $1 \mathrm{~mm}$ and the channel width is $80 \mu \mathrm{m}$. The shaded area inside the dashed box is the area that is under pump light. Right: A typical lasing spectrum of the ring dye laser.

shaded area in Fig. 12, we observed a clear multimode lasing spectrum. Lasing only occurred at the long-wavelength tail of the gain spectrum because only a small portion of the ring was pumped and the large self-absorption along the ring prevented lasing in the short-wavelength region. This structure can be used as a biosensor without any modification, in which the analytes are either functionally attached to the channel wall or simply delivered into the liquid flow and the shift in lasing wavelength is detected.

In general, the experiences and lessons from the conventional dye laser [15] and semiconductor laser communities [39] provide good guidelines for the future development of optofluidic dye lasers. For example, the development of the high-speed dye jet enabled stable continuous-wave $(\mathrm{CW})$ dye laser operation [40]. A similar device on a microfuidic chip may be needed for CW optofluidic dye lasers. Also, we should look at the various Q-switching and mode-locking methods used in conventional ultrashort pulse dye lasers and consider their possible implementations on a microfluidic platform. For one, the previously shown ring-resonator geometry is a good candidate for passive-mode locking when a suitable saturable absorber dye is introduced at the proper location on the ring.

In order to show the full potential of optofluidic dye lasers, the demonstration of a complete lab-on-a-chip system integrated with an on-chip liquid dye laser is essential. Important examples in this class include fluorescence-actuated cell sorters (FACS) [41], fluorescence-based automated Sanger DNA sequencers [42], and Raman spectrometers. Of course, we believe many other applications and novel devices that never existed before will start appearing due to the powerful optofluidic integration and adaptation.

\section{CONCLUSION}

We have demonstrated a novel microfabricated liquid DFB dye laser on a monolithic PDMS chip. Stable single-mode operation was obtained using a phase-shifted higher order Bragg gating embedded in a single-mode microfluidic channel waveguide. Its wavelength can be continuously tuned over a large range with a simple mechanical deformation method. Due to the higher order of the DFB grating in our devices, a single laser is capable of generating tunable output covering the spectral region from near ultraviolet to near infrared. Preliminary work 
on fluidic tuning showed promising results and monolithic integration with PDMS microfluidics was achieved. An integrated multispectral DFB laser array was also demonstrated by replica molding. Such laser arrays can be used to make highly parallel multiplexed biosensors and scanning-less spectrometers on a chip. It is important to note that these lasers are still not stand-alone devices because an external gas pressure source and an external pump laser are required. The gas pressure source can be eliminated by using electrokinetically-driven flow, whereas an external pump light will continue to be necessary for our dye laser designs. For portable and inexpensive devices, visible semiconductor lasers or bright LEDs can be used as the pump source.

\section{ACKNOWLEDGMENT}

The authors would like to thank Prof. A. Scherer, Z. Zhang, and T. Emery for their collaboration on this project.

\section{REFERENCES}

[1] D. Psaltis, S. R. Quake, and C. H. Yang, "Developing optofluidic technology through the fusion of microfluidics and optics," Nature, vol. 442, no. 27, pp. 371-386, 2006.

[2] Y. N. Xia and G. M. Whitesides, "Soft lithography," Annu. Rev. Mater. Sci., vol. 28, pp. 153-184, 1998.

[3] M. A. Unger, H. P. Chou, T. Thorsen, A. Scherer, and S. R. Quake, "Monolithic microfabricated valves and pumps by multilayer soft lithography," Science, vol. 288, pp. 113-116, 2000.

[4] C. L. Hansen, M. O. Sommer, and S. R. Quake, "Systematic investigation of protein phase behavior with a microfluidic formulator," Proc. Natl. Acad. Sci. USA, vol. 101, no. 40, pp. 14431-14436, Oct. 2004.

[5] T. Thorsen, S. J. Maerkl, and S. R. Quake, "Microfluidic large scale integration," Science, vol. 298, pp. 580-584, 2002.

[6] B. Helbo, A. Kristensen, and A. Menon, "A micro-cavity fluidic dye laser," J. Micromech. Microeng., vol. 13, no. 2, pp. 307-311, 2003.

[7] D. V. Vezenov, B. T. Mayers, R. S. Conroy, G. M. Witesides, P. T. Snee, Y. Chan, D. G. Nocera, and M. G. Bawendi, "A low-threshold, highefficiency microfluidic waveguide laser," J. Amer Chem. Soc., vol. 127, no. 25 , pp. 8952-8953, 2005.

[8] C. Galas, J. Torres, M. Belotti, and Q. Kou, "Microfluidic tunable dye laser with integrated mixer and ring resonator," Appl. Phys. Lett., vol. 86, pp. 264101-264103, Jun. 2005.

[9] Z. Y. Li, Z. Zhang, T. Emery, A. Scherer, and D. Psaltis, "Single mode optofluidic distributed feedback dye laser," Opt. Express, vol. 14, no. 2, pp. 696-701, Jan. 2006, [Online]. Available: http://www. opticsinfobase.org/abstract.cfm?URI=oe-14-2-696

[10] Z. Y. Li, Z. Zhang, A. Scherer, and D. Psaltis, "Mechanically tunable optofluidic distributed feedback dye laser," Opt. Express, vol. 14, no. 22, pp. 10494-10499, Oct. 2006, [Online]. Available: http://www.opticsinfobase.org/abstract.cfm?URI=oe-14-22-10494

[11] H. Kogelnik and C. V. Shank, "Stimulated emission in a periodic structure," Appl. Phys. Lett., vol. 18, no. 4, pp. 152-154, Feb. 1971.

[12] M. Nakamura, A. Yariv, H. W. Yen, S. Somekh, and H. L. Garvin, "Optically pumped GaAs surface laser with corrugation feedback," Appl. Phys. Lett., vol. 22, no. 10, pp. 515-516, May 1973.

[13] S. Baslslev and A. Kristensen, "Microfluidic single-mode laser using high-order Bragg grating and antiguiding segments," Opt. Express, vol. 13, pp. 344-351, 2005. [Online]. Available: http://www. opticsexpress.org/abstract.cfm?URI=OPEX-13-1-344

[14] A. Yariv, Optical Electronics in Modern Communications. New York, Oxford, 1997, ch. 16.

[15] F. P. Schafer, Ed., Dye Lasers (Topics in Applied Physics), vol. 1, 3rd ed. New York: Springer-Verlag, 1990.

[16] L. A. Wellerbrophy and D. G. Hall, "Analysis of wave-guide gratingsapplication of Rouard's method," J. Opt. Soc. Amer A, Opt. Image Sci., vol. 2, no. 6, pp. 863-871, Jun. 1985.

[17] S. C. De Rosa, L. A. Herzenberg, L. A. Herzenberg, and M. Roederer, "11-color, 13-parameter flow cytometry: Identification of human naive
T cells by phenotype, function, and T-cell receptor diversity," Nat. Med., vol. 7, no. 2, pp. 245-248, Feb. 2001.

[18] W. Streifer, D. R Scifres, and R. D. Burnham, "Coupling coefficients for distributed feedback single- and double-heterostructure diode lasers," IEEE J. Quantum Electron., vol. QE-11, no. 11, pp. 867-873, Nov. 1975.

[19] W. Streifer, R. D. Burnham, and D. R. Scifres, "Radiation losses in distributed feedback lasers and longitudinal mode selection," IEEE J. Quantum Electron., vol. QE-12, no. 11, pp. 737-739, Nov. 1976.

[20] J. N. Lee, C. Park, and G. M. Whitesides, "Solvent compatibility of poly(dimethylsiloxane)-based microfluidic devices," Anal. Chem., vol. 75, no. 23, pp. 6544-6554, 2003.

[21] E. P. Ippen, C. V. Shank, and A. Dienes, "Rapid photobleaching of organic laser dyes in continuously operated devices," IEEE J. Quantum Electron., vol. QE-7, no. 4, pp. 178-179, Apr. 1971.

[22] D. B. Wolfe, R. S. Conroy, P. Garstecki, B. T. Mayers, M. A. Fischbach, K. E. Paul, M. Prentiss, and G. M. Whitesides, "Dynamic control of liquid-core/liquid-cladding optical waveguides," Proc. Natl. Acad. Sci. USA, vol. 101, no. 34, pp. 12434-12438, Aug. 2004.

[23] C. V. Shank, J. E. Bjorkholm, and H. Kogelnik, "Tunable distributedfeedback dye laser," Appl. Phys. Lett., vol. 18, pp. 395-396, 1971.

[24] J. L. Wilbur, R. J. Jackman, G. M. Whitesides, E. L. Cheung, L. K. Lee, and M. G. Prentiss, "Elastomeric optics," Chem. Mater., vol. 8, no. 7, pp. 1380-1385, Jul. 1996.

[25] J. C. McDonald and G. M. Whitesides, "Poly(dimethylsiloxane) as a material for fabricating microfluidic devices," Acc. Chem. Res., vol. 35, no. 7, pp. 491-499, 2002.

[26] RTV 615 Data Sheet, GE Advanced Materials Silicones. Wilton, CT, 1998.

[27] V. Jayaraman, Z. M. Chuang, and L. A. Coldren, "Theory, design, and performance of extended tuning range semiconductor-lasers with sampled gratings," IEEE J. Quantum Electron., vol. 29, no. 6, pp. 1824-1834, Jun. 1993.

[28] Y. Oki, S. Miyamoto, M. Maeda, and N. J. Vasa, "Multiwavelength distributed-feedback dye laser array and its application to spectroscopy," Opt. Lett., vol. 27, no. 14, pp. 1220-1222, 2002.

[29] H. Po, J. D. Cao, B. M. Laliberte, R. A. Minns, R. F. Robinson, B. H. Rockney, R. R. Tricca, and Y. H. Zhang, "High power neodymium-doped single transverse mode fibre laser," Electron. Lett., vol. 29, no. 17, pp. 1500$1501,1993$.

[30] S. L. McCall, A. F. Levi, R. E. Slusher, S. J. Pearton, and R. A. Logan, "Whispering-gallery mode microdisk lasers," Appl. Phys. Lett., vol. 60, pp. 289-291, 1992.

[31] J. P. Hohimer, D. C. Craft, G. R. Hadley, G. A. Vawter, and M. E. Warren, "Single-frequency continuous-wave operation of ring resonator diode lasers," Appl. Phys. Lett., vol. 59, pp. 3360-3362, 1991.

[32] D. K. Armani, T. J. Kippenberg, S. M. Spillane, and K. J. Vahala, "Ultrahigh-Q toroid microcavity on a chip," Nature, vol. 421, pp. 925-928, 2003.

[33] S. X. Qian, J. B. Snow, H. M. Tzeng, and R. K. Chang, "Lasing dropletshighlighting the liquid-air interface by laser emission," Science, vol. 231, pp. $486-488,1986$.

[34] J. Scheuer, W. M. J. Green, G. A. DeRose, and A. Yariv, "InGaAsP annular Bragg lasers: Theory, applications, and modal properties," IEEE J. Sel. Topics Quantum Electron., vol. 11, no. 2, pp. 476-484, Mar.-Apr. 2005.

[35] O. Painter, R. K. Lee, A. Scherer, A. Yariv, J. D. O' Brien, P. D. Dapkus, and I. Kim, "Two-dimensional photonic band-gap defect mode laser," Science, vol. 284, no. 5421, pp. 1819-1821, Jun. 1999.

[36] R. K. Chang, Ed., Optical Processes in Microcavities. Singapore: World Scientific, 1996.

[37] H. Yokoyama, "Physics and device applications of optical microcavities," Science, vol. 256, no. 5053, pp. 66-70, Apr. 1992.

[38] Y. Yamamoto and R. E. Slusher, "Optical processes in microcavities," Phys. Today, vol. 46, no. 6, pp. 66-73, Jun. 1993.

[39] L. A. Coldren and S. W. Corzine, Diode Lasers and Photonic Integrated Circuits. New York: Wiley-Interscience, 1995.

[40] P. K. Runge and R. Rosenberg, "Uncofined flowing-dye films for CW dye lasers," IEEE J. Quantum Electron., vol. QE-8, no. 12, pp. 910-911, Dec. 1972.

[41] A. Y. Fu, H. P. Chou, C. Spence, F. H. Arnold, and S. R. Quake, "An integrated microfabricated cell sorter," Anal. Chem., vol. 74, pp. 2451$2457,2002$.

[42] L. M. Smith, J. Z. Sanders, R. J. Kaiser, P. Hughes, C. Dodd, C. R. Connell, C. Heiner, S. B. Kent, and L. E. Hood, "Fluorescence detection in automated DNA sequence analysis," Nature, vol. 321, no. 6071, pp. 674-679, Jun. 1986. 


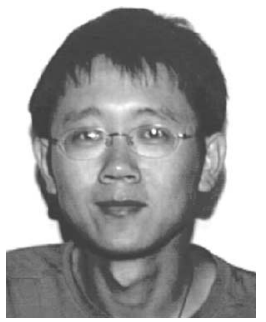

Zhenyu Li (S'06) received the B.S. degree in optics from Tsinghua University, Beijing, China, in 1999, and the M.S. degree in electrical engineering from the University of California, Santa Barbara, in 2001. $\mathrm{He}$ is currently working toward the Ph.D. degree in electrical engineering at the California Institute of Technology, Pasadena.

His current research interests include holographic information processing, optofluidic dye lasers, and integration of nanophotonics with micro/nanofluidics.

$\mathrm{Mr}$. Li is a student member of the Optical Society of America (OSA) and the Society of Photo-Optical Instrumentation Engineers (SPIE).

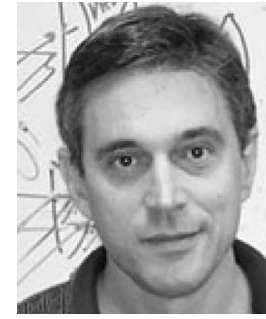

Demetri Psaltis (S'77-M'77-SM'91-F'05) received the B.S. degrees in electrical engineering and economics in 1974 and the M.S. and Ph.D. degrees in electrical engineering in 1975 and 1977, respectively, from Carnegie Mellon University, Pittsburg, PA.

In 1980, he joined the faculty at the California Institute of Technology (Caltech), Pasadena, where he is now the Thomas G. Myers Professor of Electrical Engineering. From 1992 to 1996, he was an Executive Officer in the Department of Computation and Neural Systems. From 1996 to 1999, he was the Director of the National Science Foundation Research Center on Neuromorphic Systems Engineering at Caltech. Currently, he is the Director of the Defense Advanced Research Projects Agency (DARPA) Center for Optofluidic Integration at Caltech. His current research interests include optical information processing, holography, nonlinear optics, and nanophotonics. He has authored or coauthored over 400 publications in these areas. He is the Chairman and Co-Founder of Ondax, Inc., Monrovia, CA.

Prof. Psaltis is a Fellow of the Optical Society of America and the Society of Photo-Optical Instrumentation Engineers. He is the recipient of the International Commission of Optics Prize in 1989, the Humboldt Award in 2003, and the Dennis Gabor Award in 2006. 